\title{
Atmospheric Characterization of Cold Exoplanets Using a 1.5-m Space Coronagraph
}

\author{
Anne-Lise Maire ${ }^{1}$, Raphaël Galicher ${ }^{2}$, Anthony Boccaletti ${ }^{1}$,

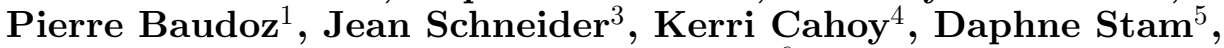 \\ and Wesley Traub 6 \\ ${ }^{1}$ LESIA, Obs. Paris/CNRS/Univ. Paris 6/Univ. Paris 7, 92195 Meudon, France
} email: Anne-Lise.Maire@obspm.fr; ${ }^{2}$ Herzberg Institute of Astrophysics, NRCC, Victoria, BC V9E 2E7, Canada; ${ }^{3}$ LUTH, Obs. Paris/CNRS/Univ. Paris 7, 92195 Meudon, France; ${ }^{4}$ Dept. of

Aeronautics and Astronautics, MIT, Cambridge, MA 02139, USA; ${ }^{5}$ SRON, Utrecht, The

Netherlands; ${ }^{6}$ JPL, CalTech, Pasadena, CA 91109, USA

\begin{abstract}
We present numerical results of the science performance of the SPICES mission, which aims to characterize the spectro-polarimetric properties of cold exoplanets and circumstellar disks in the visible. We focus on the instrument ability to retrieve the spectral signatures of molecular species, clouds and surface of super-Earths in the habitable zone of solar-type stars. Considering realistic reflected planet spectra and instrument limitation, we show that SPICES could analyse the atmosphere and surface of a few super-Earths within 5 pc of the Sun.
\end{abstract}

Keywords. planetary systems, techniques: high angular resolution, techniques: spectroscopic, methods: numerical

\section{The SPICES mission}

Direct imaging is currently the only available method to characterize the atmosphere of long-period planets, from a few tens to a few hundreds of AU. A first generation of instruments dedicated to the detection and spectral characterization in the near-infrared of young and/or massive gas giant planets is on the sky or in integration (P1640, HiCIAO, SPHERE, GPI). They aim to obtain contrasts of $10^{6}$ to a few $10^{8}$ at $<1^{\prime \prime}$. Nevertheless, the study of lighter and older planets (ice giants, super-Earths and Earths) require the development of new instruments to reach contrasts 100 to 1000 times higher $\left(10^{8}-10^{10}\right)$. Extremely Large Telescopes $(30-40 \mathrm{~m})$ may allow the detection of ice giants and superEarths around a large sample of targets in the near-infrared. However, spectroscopy of these planets will be very challenging due to the Earth atmosphere (turbulence, absorption in spectral lines of interest). Meanwhile, projects of small ( $\sim 1.5$-m diameter $)$ space coronagraphs observing in the visible are studied in order to address these questions.

SPICES (Spectro-Polarimetric Imaging and Characterization of Exoplanetary Systems) belongs to this family of projects and was submitted to the ESA Cosmic Vision call for medium-class missions in 2010 by a consortium of European, American and Japanese institutes (see Boccaletti et al., this volume and Boccaletti et al. 2012). To date, studies on small space coronagraphs have focused on the validation of technologies, such as coronagraphy, wavefront correction and differential imaging, to attenuate the stellar photon noise and quasistatic aberrations induced by the optical defects of the instrument. However, the assessment of their science performance has been largely unexplored in detail so far. We discuss here the SPICES ability to retrieve the spectral signatures of molecular species, clouds and surface of super-Earths in the habitable zone of solar-type stars, following the topic of this volume. We refer the reader to Maire et al. (2012) for a detailed description of the methods and of other science cases (gas and ice giant planets). 
Table 1. Parameters of the model spectra of Earth-like planets used for our study.

\begin{tabular}{|c|c|c|c|c|}
\hline Separation $[\mathbf{A U}]$ & Radius $\left[\mathbf{R}_{\mathrm{E}}\right]$ & Atmospheric structure & Surface type \\
\hline 1 & 2.5 & 0,50 and $100 \%$ clouds & Forest, ocean and forest-ocean mix \\
\hline
\end{tabular}

\section{Numerical model of the instrument and hypotheses}

To achieve contrasts as high as $10^{9}-10^{10}$ at a few tenths of an arcsecond, SPICES requires a specific instrumentation, which is not available on any other present or future facility. It comprises a 1.5-m high-quality off-axis telescope, high-accuracy wavefront control, a broad-band coronagraph with small inner working angle (i.e., the angular separation of $50 \%$ throughput), and optical elements and detectors to collect the polarimetric and spectroscopic information. We developed a numerical model of the instrument which simulates the critical subsystems: the primary mirror, the deformable mirror, the coronagraph and the wavefront sensor. The spectral bandwidth is $0.45-0.9 \mu \mathrm{m}$ and is split into 35 channels of the same spectral width (mean spectral resolution $\mathrm{R}=50$ ). We account for a realistic overall transmission of the instrument $(\sim 16 \%)$, photon noise (from the star, the zodiacal light and an exozodiacal cloud of the same intensity of the Solar System cloud), read-out noise of the detector and variations of the response of the detector pixels.

To assess the science performance, we used model spectra of Earth-like planets (Stam 2008, and Table 1). These models are calculated assuming planets at 1 AU from a solartype star for several cloud coverage and surface type. We defined a characterization criterion based on the signal-to-noise ratio (SNR) of the spectral measurements. We derived that SNR of at least 25 and 30 are required to identify the cloud coverage for a given surface and the surface type for a cloud coverage of $50 \%$, respectively. We finally calculated the exposure time to meet these requirements. We discuss below some results.

\section{Retrieval of spectral properties of super-Earths}

Figure 1 shows the spectra measured from our simulations (symbols with error bars) and the corresponding theoretical spectra (solid lines) for the analysis of the cloud coverage (left) and the surface type (right). For the first science case, the SNR constraint of 25 is satisfied in $150 \mathrm{~h}$ at the maximum star distance for which the star-planet separation is resolved by SPICES. Systems closer than $5 \mathrm{pc}$ will also be accessible for the characterization of the cloud coverage. The influence of clouds is more important in the blue, whereas the red wavelengths are dominated by the strong reflection of the vegetation (left). The main atmospheric gases $\left(\mathrm{O}_{2}\right.$ at $0.76 \mu \mathrm{m}$ and $\mathrm{H}_{2} \mathrm{O}$ at 0.72 and $\left.0.82 \mu \mathrm{m}\right)$ are quite well retrieved whatever the cloud coverage (the measured SNR are $>5$ and $>15$, respectively). The broad ozone signature $(\sim 0.5-0.7 \mu \mathrm{m})$ is mostly detectable when the cloud coverage is large (the measured SNR is $\sim 23$ ). As for the analysis of the surface type (right), an integration time of $200 \mathrm{~h}$ is necessary to reach a measured SNR of 30 for a star distance of $4 \mathrm{pc}$. The differences between surface types are larger in the half red part of the spectra. $\mathrm{O}_{2}$ and $\mathrm{H}_{2} \mathrm{O}$ bands as well as the "red edge" can still be measured.

Finally, we studied the space volume of targets accessible to our instrument in terms of planet radius and star distance for three planet types (gas and ice giants, Earths, Cahoy et al. 2010; Stam 2008). For this, we assumed that the planet properties do not change with the radius for the ranges we consider $\left(5.5 R_{E} \leqslant R_{p} \leqslant 12 R_{E}, 2 R_{E} \leqslant R_{p} \leqslant 6 R_{E}\right.$ and $R_{p} \leqslant 2.5 R_{E}$, respectively). This is shown in Figure 2 for a solar-type star (left) and an M0 star (right). Since the model spectra we used are calculated for discrete separations from a Sun analog, we assumed flux conservation to derive the corresponding separations 

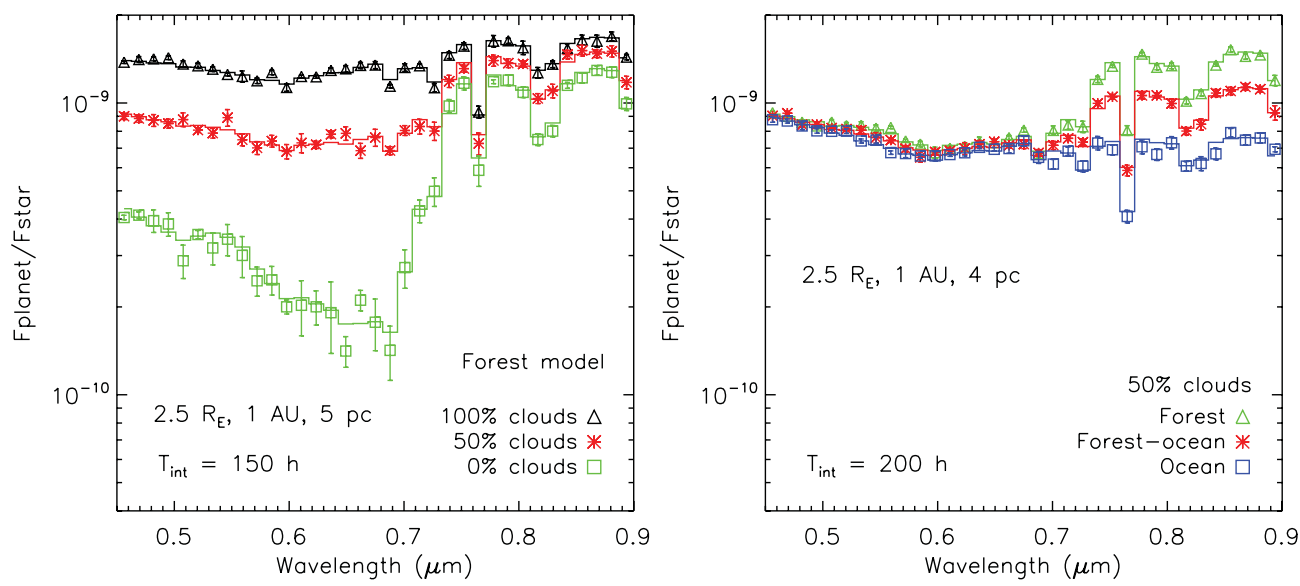

Figure 1. Theoretical (solid lines) and simulated (symbols) spectra of $2.5 R_{\mathrm{E}}$ Earth-like planets for different cloud coverage (left) and surface type (right). Error bars show the $1-\sigma$ dispersion of the measurements for five noise realizations.
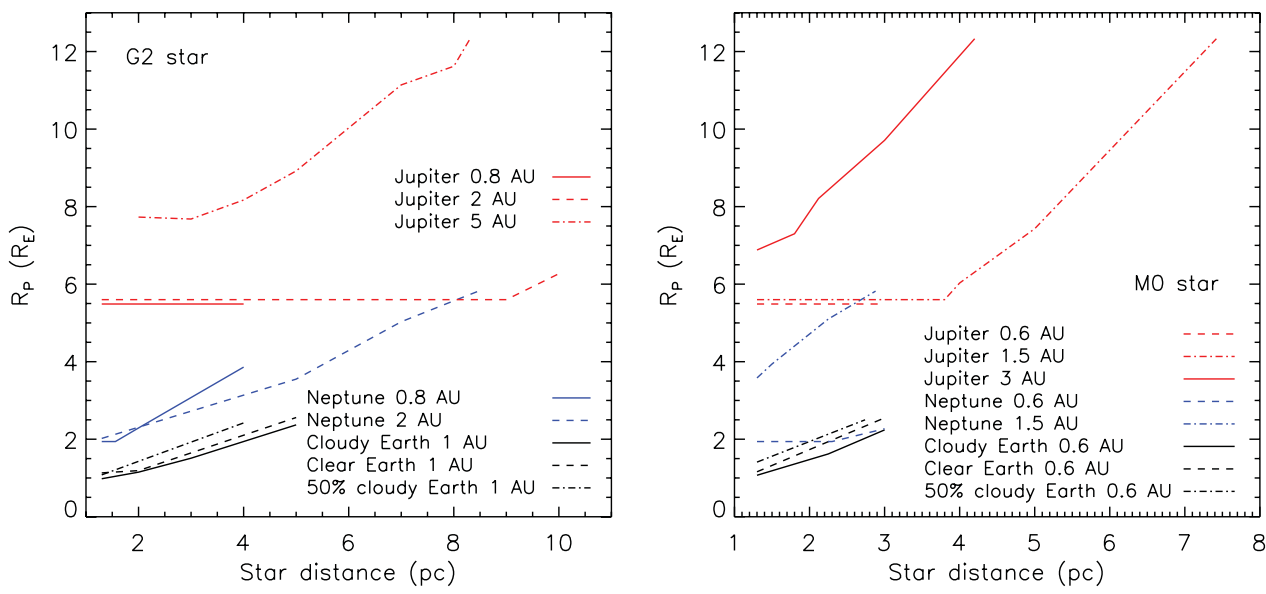

Figure 2. Minimum radius of model planets for which characterization is possible (measured SNR of 12-30) as a function of the star distance for a G2 star (left) and a M0 star (right).

from an M0 star (except for the Earths, for which we extrapolate the 1-AU model to a separation of $2 \mathrm{AU}$ ). From these results, we estimated that $\sim 300$ potential planetary systems could be analyzed, including a dozen of stars for characterization of super-Earths.

\section{Conclusion}

The identification of the main properties of reflected-light spectra of super-Earths (molecules, clouds, surface) requires signal-to-noise ratios of at least 30. A 1.5-m space coronagraph could achieve this value for a dozen of super-Earths within $5 \mathrm{pc}$ of the Sun.

\section{References}

Boccaletti, A., Schneider, J., Traub, W., et al. 2012, Exp. Astron., 34, 355

Cahoy, K. L., Marley, M. S, \& Fortney, J. J. 2010, ApJ, 724, 189

Maire, A.-L., Galicher, R., Boccaletti, A., et al. 2012, A\&A, 541, A83

Stam, D. 2008, A\&AA, 482, 989 